\title{
Ejection-collision orbits in the RTBP
}

\author{
Mercè Olléa $^{\mathrm{a}}$ Òscar Rodríguez ${ }^{\mathrm{a}}$, Jaume Soler $^{\mathrm{b}}$ \\ ${ }^{a}$ Dept. Matemàtiques. Universitat Politècnica de Catalunya, Av Diagonal 647, 08028 \\ Barcelona, Spain. \\ ${ }^{b}$ Dept. d'Enginyeria Civil i Ambiental. Universitat Politècnica de Catalunya, Av Diagonal \\ 647, 08028 Barcelona, Spain.
}

\begin{abstract}
In this paper we analyse the ejection-collision (EC) orbits of the planar restricted three body problem. Being $\mu \in(0,0.5]$ the mass parameter, and taking the big (small) primary with mass $1-\mu(\mu)$, an EC orbit will be an orbit that ejects from the big primary, does an excursion and collides with it. As it is well known, for any value of the mass parameter $\mu \in(0,0.5]$ and sufficiently restricted Hill regions (that is, for big enough values of the Jacobi constant $C$ ), there are exactly four EC orbits. We check their existence and extend numerically these four orbits for $\mu \in(0,0.5]$ and for smaller values of the Jacobi constant. We introduce the concept of $n$-ejection-collision orbits ( $n$-EC orbits) and we explore them numerically for $\mu \in(0,0.5]$ and values of the Jacobi constant such that the Hill bounded possible region of motion contains the big primary and does not contain the small one. We study the cases $1 \leq n \leq 10$ and we analyse the continuation of families of such $n$-EC orbits, varying the energy, as well as the bifurcations that appear.
\end{abstract}

Keywords: regularization, ejection-collision orbits, invariant manifolds, bifurcations.

2010 MSC: 70F07, 70F15

Email addresses: Merce.0lle@upc.edu (Mercè Ollé), orodriguezdelrio@gmail.com (Òscar Rodríguez), jaume.soler.villanueva@upc.edu (Jaume Soler) 


\section{Introduction}

We consider the restricted three-body problem (RTBP), which consists of the description of the motion of an infinitesimal body $P$ under the attraction of two bodies called primaries, $P_{1}$ and $P_{2}$ of masses $m_{1}=1-\mu$ and $m_{2}=\mu$, for

$5 \mu \in(0,0.5]$, which describe circular orbits around their common center of mass located at the origin. In particular, a solution of the RTBP has a collision with the big primary $P_{1}$ at the instant $t_{0}$ if the distance between the particle and $P_{1}$ is equal to 0 at $t_{0}$. We will distinguish between an ejection orbit (that is, the particle kicks out from collision with $P_{1}$ at some instant $t_{0}$ ) and a collision orbit (the particle goes to collision with $P_{1}$ at some instant $t_{1}$ ). An orbit defined on the interval $\left[t_{0}, t_{1}\right]$ is called an ejection-collision orbit if it has an ejection at $t=t_{0}$ and a collision at $t=t_{1}$. This paper is devoted to the study of ejection-collision (EC) orbits.

Regarding analytical studies of EC orbits in the planar RTBP, Llibre [10] proved the existence of at least two EC orbits for $\mu>0$ small enough and the energy $H$ small enough (or equivalently the Jacobi constant $C$ big enough). In Lacomba and Llibre [9] the authors used the existence of such transversal EC orbits to prove that both the Hill problem and the RTBP have no $C^{1}$-extensible regular integrals. Chenciner and Llibre [6] proved the existence of four EC orbits for any value of $\mu \in(0,0.5]$ and $H$ small enough. Concerning the spatial RTBP Llibre and Martinez Alfaro [11] extended the existence of EC orbits for small enough values of the mass parameter. Finally in [12] and [15], the authors considered the planar elliptic RTBP and proved the existence of EC orbits for both the mass parameter and the eccentricity small enough. We remark that such results are obtained mainly applying blow up techniques (see [13] and [10] for the original ideas), regularization of variables (the specific changes of variables used are due to [10] but the regularization theory applied to Celestial Mechanics can also be found in [19] and [18]) and the perturbation approach for these particular problems can be found in the aforementioned references.

Referring to numerical results, we mention Henon's paper about the compu- 
tation of EC orbits obtained along the continuation of some families of symmetric periodic -non-collision- orbits in the Copenhagen problem (that is $\mu=0.5$, see [7]) and also for Hill's problem (see [8]). Finally, the evolution of 16 particular collision periodic orbits obtained from the $\mu=0.5$ case was numerically 35 studied for various values of the mass ratio $\mu$ in [4].

Concerning realistic models of concrete physical systems, the role of the EC orbits (and orbits close to them) is relevant not only for astronomic problems: in order to determine regions of capture of irregular moons by giant planets (see [1]), or to explain the formation of Kuiper-belt binaries through physical 40 collisions between the binary and further intruders (see [2]) but also for microscopic scale problems: for example, in the hydrogen atom subject to a linear or circularly polarized microwave field, the collisions between the electron and the core are a dominant ionization mechanism (see a discussion in [5] and [14]).

One of the reviewers drew our attention to the paper [17] on disruption of rubble-pile asteroids which are modelled as a loose aggregate of small boulders: numerical simulations show that under some conditions a number of the boulders are shed off the main body, prior to the disruption of the asteroid. The motion of an ejected boulder will be close to an ejection orbit if its angular momentum immediately after the ejection is close to zero. Although the rotation of the main 50 body means the system has a certain angular momentum, the ejected piece need not take away a lot of it onto its orbit because most of the angular momentum will stay with the rotating rubble-pile. It seems however that a closer look at the mechanism of shedding should be taken if the model of ejection-collision orbits were to be considered. On the other hand, a number of asteroids are known to have a tiny satellite which could be modelled as the small primary, and the other characteristic feature of asteroids, namely their being far from sphere-shaped, would lead to a more complicated expression for the gravitational potential and probably a different behaviour of the ejection orbits but not to insuperable numerical difficulties.

In this paper we focus on what we call $n$-ejection-collision orbits, defined as EC orbits such that eject from the big primary $P_{1}$, reach $n$ times a relative 
maximum in the distance with respect to $P_{1}$ and finally collide with it. The approach is numerical and our goals are twofold: (i) On the one hand, we concentrate on 1-EC orbits. (i.1) We verify the existence of four 1-EC orbits for any value of $\mu>0$ and very small values of $H$ (known analytical results), (i.2) we extend these results for any value of the mass parameter and less restrictive values of the energy obtaining families of 1-EC orbits when varying the energy for a fixed $\mu$ and (i.3) we analyse some bifurcations which appear. (ii) On the other hand, we make a similar study for $n$-EC orbits, with $n>1$. As far as we know, there are no published results concerning parts (i.2), (i.3) and (ii).

The paper is divided as follows: Section 2 is devoted to a short description of the RTBP and some notation is introduced. In order to deal with the singularity arising from the zero distance between $P$ and $P_{1}$, we recall McGehee's ideas to regularize the equations of motion which become regular when $P$ is at $P_{1}$.

In Section 3 we describe the collision manifold, for any value of the mass parameter and we regard EC orbits as heteroclinic connections between different equilibrium points living on the collision manifold.

In Section 4 we focus on the $n$-EC orbits. We explain the numerical methodology used and describe the results for $1 \leq n \leq 5$. Finally we draw some conclusions.

\section{The RTBP}

The circular, restricted three-body problem (RTBP) describes the motion of a particle of infinitesimal mass, moving under the gravitational influence of two massive bodies, called primaries, that describe circular orbits around their common center of mass. We will consider the planar RTBP, in which the motion of the particle is contained in the plane of motion of the primaries. Taking a coordinate system that rotates with the primaries, with origin located at their center of mass, and suitable units, we can assume that the primaries have masses $1-\mu$ and $\mu, \mu \in(0,0.5]$, their positions are fixed at $(-\mu, 0)$ and $(1-\mu, 0)$, respectively, and the period of their motion is $2 \pi$. With these assumptions, 
the equations of motion for the particle in this rotating (also called synodical system) are given by

$$
\begin{aligned}
& \ddot{x}-2 \dot{y}=\frac{\partial \Omega}{\partial x}(x, y) \\
& \ddot{y}+2 \dot{x}=\frac{\partial \Omega}{\partial y}(x, y),
\end{aligned}
$$

where

$$
\Omega(x, y)=\frac{1}{2}\left(x^{2}+y^{2}\right)+\frac{1-\mu}{\sqrt{(x+\mu)^{2}+y^{2}}}+\frac{\mu}{\sqrt{(x+\mu-1)^{2}+y^{2}}}+\frac{1}{2} \mu(1-\mu) .
$$

and ${ }^{\circ}=d / d t$.

It is well known that this system of ODE has the following properties (see [19] for details) which will be used along the paper:

1. It has a first integral, the so-called Jacobi integral, defined by

$$
C=2 \Omega(x, y)-\dot{x}^{2}-\dot{y}^{2}
$$

2. The equations of motion satisfy the symmetry

$$
(t, x, y, \dot{x}, \dot{y}) \longrightarrow(-t, x,-y,-\dot{x}, \dot{y}) .
$$

This means that, given any solution $(x(t), y(t), \dot{x}(t), \dot{y}(t))$ of system (1), forward in time, there exists another solution of system (1), backwards in time, $(x(-t),-y(-t),-\dot{x}(-t), \dot{y}(-t))$. In particular, this implies that given a trajectory for the particle (in the configuration projection $(x, y)$ ), there exists another one which is symmetric with respect to the $x$ axis (see [19]). This symmetry $90 \quad$ will be observed in Section 4 .

3. There exist 5 equilibrium points (with $(\dot{x}, \dot{y})=(0,0)$ ): the collinear ones, $L_{i}, i=1,2,3$ on the $x$ axis, and the triangular ones $L_{i}, i=4,5$ located at the vertices of an equilateral triangle with the primaries. We will assume that $x_{L_{2}} \geq 1-\mu \geq x_{L_{1}} \geq-\mu \geq x_{L_{3}}$, that is, $L_{1}$ is between the primaries, $L_{2}$ is on 95 the right hand side of the small one and $L_{3}$ on the left hand side of the big one. We will denote by $C_{L_{i}}(\mu)$ the value of the Jacobi constant at $L_{i}$ for a given $\mu$. 4. The equations of motion can be written as a Hamiltonian system in position $(x, y)$ and momenta $\left(p_{x}, p_{y}\right)$ variables, with $p_{x}=x^{\prime}-y$ and $p_{y}=y^{\prime}+x$, defined 
by the Hamiltonian function

$$
H\left(x, y, p_{x}, p_{y}\right)=\frac{1}{2}\left(p_{x}^{2}+p_{y}^{2}\right)+y p_{x}-x p_{y}-\frac{1-\mu}{r_{1}}-\frac{\mu}{r_{2}}-\frac{1}{2} \mu(1-\mu)
$$

with $r_{1}=\sqrt{(x+\mu)^{2}+y^{2}}$ and $r_{2}=\sqrt{(x+\mu-1)^{2}+y^{2}}$, and the relation between $C$ and $H$ is given by

$$
H=-\frac{C}{2} .
$$

We denote by $H_{L_{i}}(\mu)$, the associated value of the Hamiltonian at $L_{i}$ for a given $\mu$.

5. Fixed a value of the Jacobi constant $C$ (or the Hamiltonian $H$ ), the motion is allowed to take place in the Hill's region defined by

$$
\mathcal{R}(C)=\left\{(x, y) \in \mathbb{R}^{2} \mid 2 \Omega(x, y) \geq C\right\} .
$$

In this paper we will restrict the range of values of $C$ to $C \geq C_{L_{2}}(\mu)(H \leq$ $H_{L_{2}}(\mu)$, see in Figure 1 the corresponding Hill's regions). Actually, along the paper, we will use specific values of $H$, that can be translated to values of $C$ through relation (5).

6. Regularization of the big primary. Since our goal is to study the ejectioncollision orbits of the big primary, a first step is to deal with the singularity appearing in the equations when $r_{1}=0$.

To do so, we make a translation so that the primary of mass $1-\mu$ is located at the origin of coordinates and that of mass $\mu$ at $(1,0)$, that is, we consider $q_{1}=x+\mu, q_{2}=y, p_{1}=p_{x}, p_{2}=p_{y}$. In this reference system the Hamiltonian becomes (using the same notation $H$ )

$$
H\left(q_{1}, q_{2}, p_{1}, p_{2}\right)=\frac{1}{2}\left(p_{1}^{2}+p_{2}^{2}\right)+p_{1} q_{2}-p_{2} q_{1}-\frac{1-\mu}{r_{1}}+\mu p_{2}-\frac{\mu}{r_{2}}-\frac{1}{2} \mu(1-\mu),
$$

with $r_{1}=\sqrt{q_{1}^{2}+q_{2}^{2}}$ and $r_{2}=\sqrt{\left(q_{1}-1\right)^{2}+q_{2}^{2}}$.

Now we introduce the canonical change of polar coordinates

$$
\begin{array}{ll}
q_{1}=r \cos \theta & p_{1}=p_{r} \cos \theta-\frac{p_{\theta}}{r} \sin \theta \\
q_{2}=r \sin \theta & p_{2}=p_{r} \sin \theta+\frac{p_{\theta}}{r} \cos \theta
\end{array}
$$




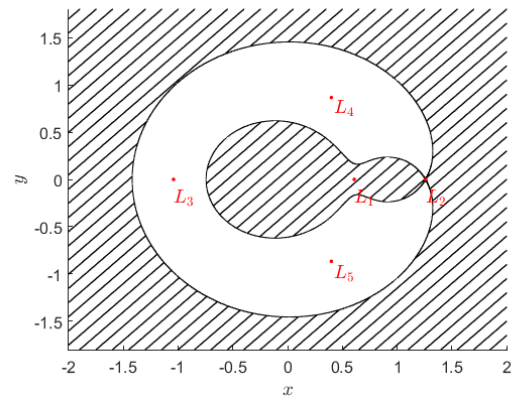

(a) $C=C_{L_{2}}$

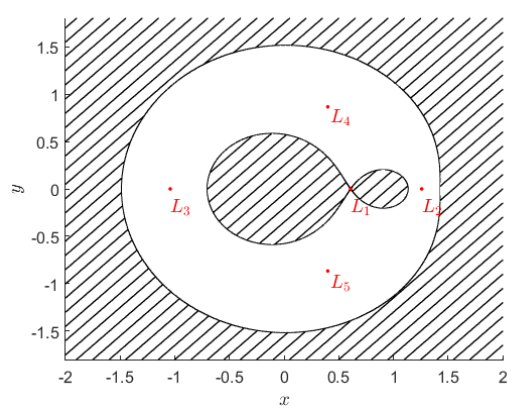

(c) $C=C_{L_{1}}$

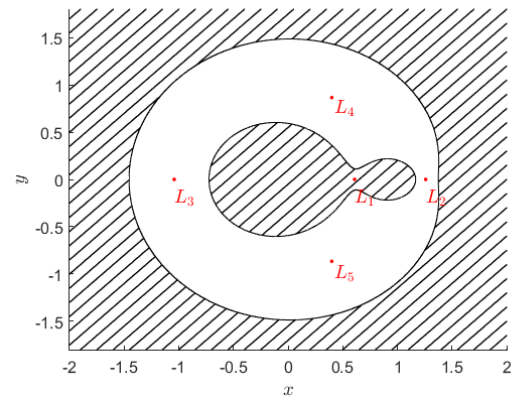

(b) $C_{L_{2}}<C<C_{L_{1}}$

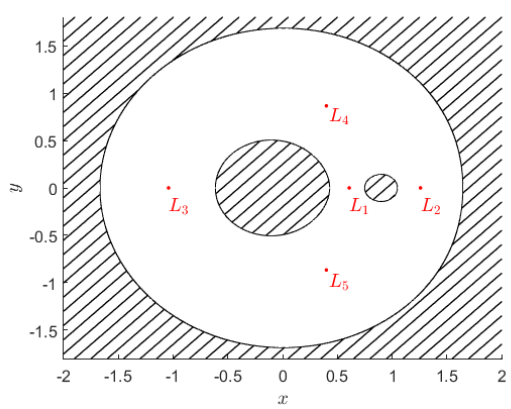

(d) $C>C_{L_{1}}$

Figure 1: Hill's region according to the Jacobi constant $C$.

and the Hamiltonian (6) reads

$$
\begin{aligned}
& H\left(r, \theta, p_{r}, p_{\theta}\right)= \\
& \quad \frac{1}{2}\left(p_{r}^{2}+\frac{p_{\theta}^{2}}{r^{2}}\right)-p_{\theta}-\frac{1-\mu}{r}+\mu\left(p_{r} \sin \theta+\frac{p_{\theta}}{r} \cos \theta\right)-\frac{\mu}{\sqrt{1+r^{2}-2 r \cos \theta}}-\frac{1}{2} \mu(1-\mu)(8)
\end{aligned}
$$

The associated Hamiltonian system of ODE is

$$
\begin{aligned}
\dot{r} & =p_{r}+\mu \sin \theta \\
\dot{\theta} & =\frac{p_{\theta}}{r^{2}}-1+\frac{\mu}{r} \cos \theta \\
\dot{p}_{r} & =\frac{p_{\theta}^{2}}{r^{3}}-\frac{1-\mu}{r^{2}}-\mu \frac{r-\cos \theta}{\left(1+r^{2}-2 r \cos \theta\right)^{3 / 2}}+\mu \frac{p_{\theta} \cos \theta}{r^{2}} \\
\dot{p}_{\theta} & =\mu\left[-\frac{r \sin \theta}{\left(1+r^{2}-2 r \cos \theta\right)^{3 / 2}}-p_{r} \cos \theta+\frac{p_{\theta}}{r} \sin \theta\right] .
\end{aligned}
$$

Applying McGehee's ideas (see [10] and [13]), we introduce the new variables 


$$
v=\dot{r} r^{1 / 2} \quad u=r^{3 / 2} \dot{\theta}
$$

and a change of time $d t / d \tau=r^{3 / 2}$, such that the system of ODE becomes

$$
\begin{aligned}
r^{\prime}= & v r \\
\theta^{\prime}= & u \\
v^{\prime}= & \frac{1}{2} v^{2}+u^{2}+2 u r^{3 / 2}+r^{3}-(1-\mu) \\
& -\mu r^{2} \cos \theta-\mu r^{2} \frac{r-\cos \theta}{\left(1+r^{2}-2 r \cos \theta\right)^{3 / 2}} \\
u^{\prime}= & -\frac{1}{2} u v-2 v r^{3 / 2}+\mu r^{2} \sin \theta\left(1-\frac{1}{\left(1+r^{2}-2 r \cos \theta\right)^{3 / 2}}\right),
\end{aligned}
$$

where $^{\prime}=d / d \tau$. We remark that the singularity $r=0$ has been removed and

this system has just one singularity - the collision with the small primary $r=1$, $\theta=0$. If we consider the hamiltonian (7) in these variables, which is a first integral, the relation $H=\mathfrak{H}$ becomes

$$
0=-r \mathfrak{H}+\frac{1}{2}\left(v^{2}+u^{2}\right)-\frac{1}{2} r^{3}-(1-\mu)+\mu r^{2} \cos \theta-\mu \frac{r}{\sqrt{1+r^{2}-2 r \cos \theta}}-\frac{1}{2} \mu r
$$

\section{The collision manifold. Ejection/collision orbits.}

System (11) has an invariant manifold $\Lambda$ defined by $r=0$, called the collision manifold. From (12) we conclude that $\Lambda$ is a torus (see Figure 2),

$$
\Lambda=\left\{u^{2}+v^{2}=2(1-\mu), \quad \theta \in[0,2 \pi]\right\}
$$

and the dynamics on this torus is given by

$$
\begin{aligned}
\theta^{\prime} & =u \\
v^{\prime} & =\frac{1}{2} v^{2}+u^{2}-(1-\mu) \\
u^{\prime} & =-\frac{1}{2} u v .
\end{aligned}
$$

We want to point out that this torus is on the boundary of each energy level 


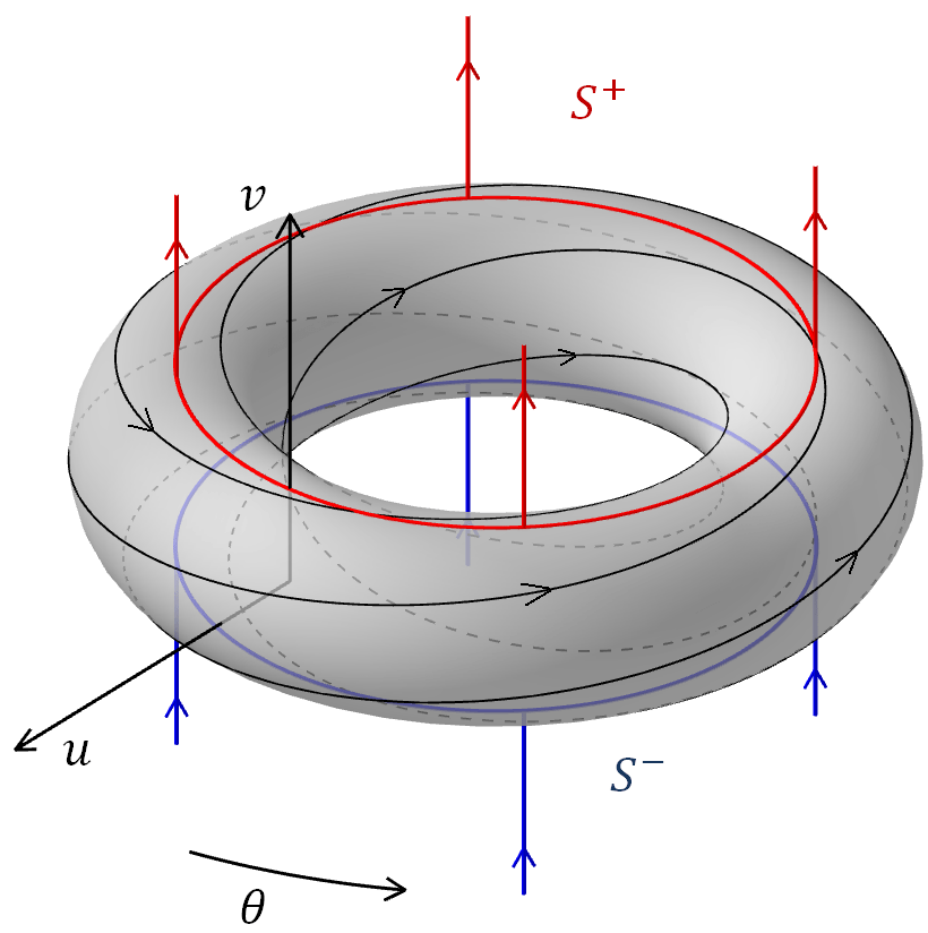

Figure 2: Collision manifold

For our purposes we remark that on $\Lambda$ there exist two circles of equilibrium points defined by $S^{+}=\left\{r=0, \theta, v=v_{0}, u=0, \theta \in[0,2 \pi]\right\}$ and $S^{-}=\{r=$ $\left.0, \theta, v=-v_{0}, u=0, \theta \in[0,2 \pi]\right\}$ with $v_{0}=+\sqrt{2(1-\mu)}$. We also observe that the tangent vector to both circles of equilibria is given by $(0,1,0,0)$.

Let us define the matrices $M^{ \pm}$as the linearization of system (11) at the corresponding equilibrium points $S^{ \pm}$; they are given by

$$
M^{ \pm}=\left(\begin{array}{cccc} 
\pm v_{0} & 0 & 0 & 0 \\
0 & 0 & 0 & 1 \\
0 & 0 & v_{0} & 0 \\
0 & 0 & 0 & \mp v_{0} / 2
\end{array}\right)
$$

The matrix $M^{+}$has eigenvalues:

$$
\lambda_{1}=-v_{0} / 2, \lambda_{2}=\lambda_{3}=v_{0}, \lambda_{4}=0
$$


and corresponding eigenvectors:

$$
\boldsymbol{v}_{1}=\left(0,-2 / v_{0}, 0,1\right), \boldsymbol{v}_{2}=(0,0,1,0), \boldsymbol{v}_{3}=(1,0,0,0), \boldsymbol{v}_{4}=(0,1,0,0)
$$

So for each equilibrium point $P \in S^{+}$we have a 2-d unstable manifold $W^{u}(P)$ and a 1-d stable one $W^{s}(P)$. Similarly, for each equilibrium point $Q \in S^{-}$, we have a 2 -d stable manifold $W^{s}(Q)$ and a 1 -d unstable one $W^{u}(Q)$.

At this point we distinguish between 3 types of orbits: (i) ejection, (ii) collision and (iii) ejection-collision orbits.

(i) The set of ejection orbits -those which are ejected from collision with the big primary- is the set of orbits on the unstable manifold $W^{u}(P)$, for any $P=\left(0, \theta, v_{0}, 0\right) \in S^{+}$. So each ejection orbit may be regarded as an orbit such that $r>0$ for all finite time $\tau$ and asymptotically tends to an equilibrium point $P \in S^{+}$as $\tau \rightarrow-\infty$.

(ii) The set of collision orbits - those which arrive at collision with the big primary - is the set of orbits on the stable manifold $W^{s}(Q)$, for any $Q=$ $\left(0, \theta,-v_{0}, 0\right) \in S^{-}$. So each collision orbit may be regarded as an orbit such that $r>0$ for all finite time $\tau$ and asymptotically tends to an equilibrium point $Q \in S^{-}$as $\tau \rightarrow+\infty$.

(iii) The set of ejection-collision orbits -those which eject from/arrive at collision with the big primary- is the set of orbits obtained from the intersection $W^{u}\left(S^{+}\right) \cap W^{s}\left(S^{-}\right)$. So they may be regarded as heteroclinic orbits between $P \in S^{+}$and $Q \in S^{-}$.

\subsection{Particular case $\mu=0$.}

In order to have a first insight of such type of orbits, let us consider the particular case $\mu=0$.

As it is well known, an ejection/collision orbit in this case is characterized by the angular momentum $M=r^{2}(d \theta / d t+1)$ equal to 0 (see [19]) that is $M=0$, or equivalently, using the $u$ variable, by $u=-r^{3 / 2}$. So, the manifold of ejection/collision orbits at the energy level $H=\mathfrak{H}$ defined by (12) now becomes 


$$
r \mathfrak{H}=\frac{1}{2} v^{2}-1
$$

We plot in Figure 3 the associated level curves and in Figure 4 we visualize the corresponding manifolds in variables $(r, \theta, v)$. In particular, for $H=\mathfrak{H}<0$ we obtain the coinciding manifolds of ejection-collision orbits. If $H=\mathfrak{H}$ is either equal to 0 or positive, we obtain the manifolds consisting of the ejectionparabolic escape orbits (and parabolic escape-collision ones) and the ejectionhyperbolic escape orbits (and hyperbolic escape-collision ones) respectively, in the sense that they arrive at (depart from) infinity with zero or positive velocity.

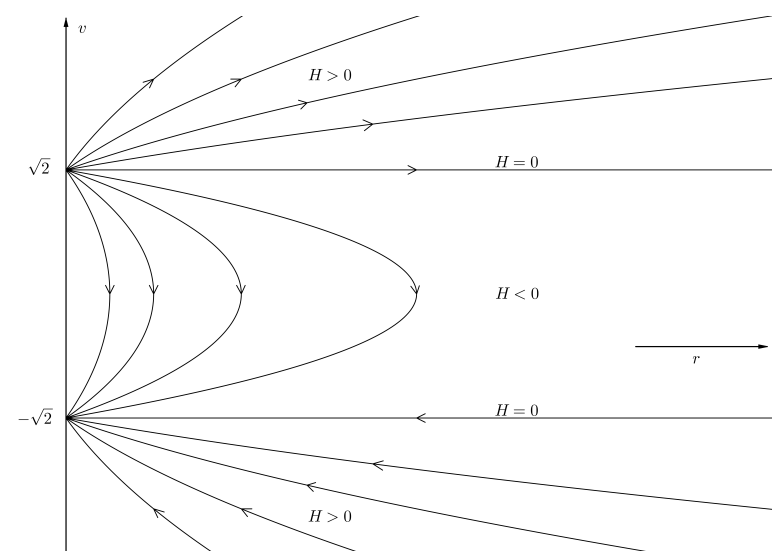

Figure 3: $\mu=0$. Level curves of $r H=\frac{1}{2} v^{2}-1$

We are particularly interested in this paper in ejection-collision orbits. We have just shown that for $\mu=0$ the ejection manifold and the collision one coincide for $H<0$. Thus any orbit ejecting from the primary ends colliding with it.

Now we want to study the general case $\mu \neq 0$ and $H<0$. When $\mu$ is different from zero, the influence of the small primary somewhat deforms the ejection and collision manifolds and these manifolds do not coincide any more. The dynamics is much richer and intricate in this case and the subject of the next Sections is to study, from a numerical point of view, this dynamics. 

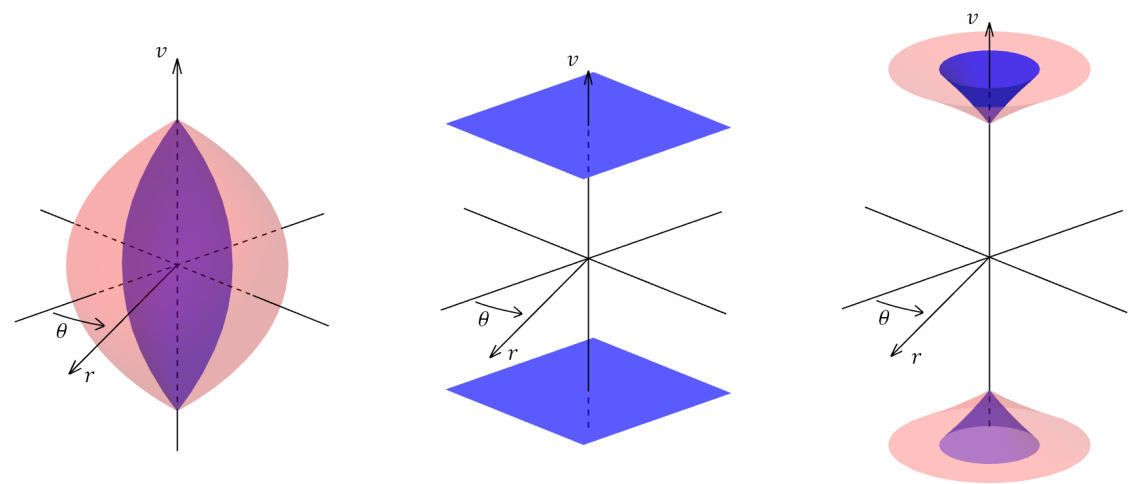

Figure 4: $\mu=0$. Ejection-collision manifold for $H<0$ (left). Ejection and collision manifolds for $H=0$ (middle) and $H>0$ (right).

\section{4. $n$-Ejection-collision orbits}

We are now focused on what we call $n$-ejection-collision orbits, simply noted by $n$-EC orbits, that is orbits that eject from the big primary and reach $n$ times a relative maximum in the distance $r$ before colliding with the big primary. See Figure 5.
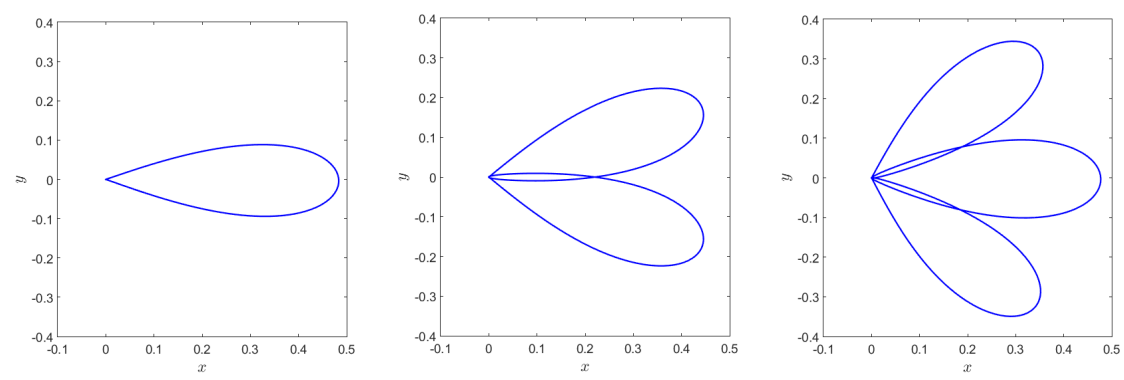

Figure 5: Examples of $n$-ejection-collision orbits for $n=1,2,3$ (from left to right). For $n=2(n=3)$, there is $1(2)$ close passages to collision between ejection and collision.

For the particular case $n=1$, it is proved analytically in [6] that, for $H=$ $\mathfrak{H}<0$ small enough and any value of $\mu \in(0,0.5]$, there exist exactly four 1 ejection-collision orbits. We remark, however, that the Hill's regions considered in that paper are very restrictive. 
In this section, we will generalise the previous results in two directions. On the one hand, concerning 1-EC orbits, we will show that these four 1-EC orbits exist for any value of the mass parameter $\mu \in(0,0.5]$ and less restrictive Hill's regions, and we analyse some bifurcations as well as the existence of some new families. On the other hand, the existence of $n$-ejection-collision orbits $(n \geq 2)$ for any value of the mass parameter and $H \leq H_{L_{1}}(\mu)$ is also analysed, families of $n$-EC orbits are computed and the appearing bifurcations are discussed.

But first let us shortly describe the numerical methodology used to deal with EC orbits.

\subsection{Numerical methodology}

We focus our attention on (i) a procedure to compute ejection (collision) orbits. (ii) To show the existence of a precise number of EC orbits for $\mu$ and $H$ given. (iii) To compute a particular $n$-EC orbit.

(i) For fixed $H=\mathfrak{H}$ and for each equilibrium point $P=\left(0, \theta_{0}, v_{0}, 0\right) \in S^{+}$, parameterized by an angle $\theta_{0} \in[0,2 \pi]$, the associated unstable manifold $W^{u}(P)$ reduces to a single orbit, which is an ejection orbit. To obtain such orbit, we just need an initial condition (that will be taken close to $P$ ) and to integrate the sytem of ODE (11) forward in time. We take as initial condition a point on the tangent direction to this orbit; more precisely

$$
\left(0, \theta_{0}, v_{0}, 0\right)+s \frac{\boldsymbol{w}}{\|\boldsymbol{w}\|}
$$

with $\boldsymbol{w}=\left(1,0,\left(\mathfrak{H}+\frac{3}{2} \mu\right) / v_{0}, 0\right)$ and $s>0$ a small quantity (typically $\left.10^{-6}\right)$. Let us observe that the specific vector $\boldsymbol{w}$ is obtained by the restriction that the normal vector to this energy level set at point $P=\left(0, \theta_{0}, v_{0}, 0\right)$, which is $\boldsymbol{n}=\left(-\mathfrak{H}-\frac{3}{2} \mu, 0, v_{0}, 0\right)$, must be perpendicular to the tangent plane passing through $P$ and generated by the eigenvectors $\boldsymbol{v}_{2}$ and $\boldsymbol{v}_{3}$ (see Section 3), i.e. vectors like $\boldsymbol{v}=(\beta, 0, \gamma, 0)$, with $\beta, \gamma \in \mathbb{R}$. So the condition $\beta\left(-\mathfrak{H}-\frac{3}{2} \mu\right)+\gamma v_{0}=0$ must be satisfied.

Varying $\theta_{0} \in[0,2 \pi]$, we generate a set of initial conditions and the corresponding ejection orbits. So we have $W^{u}\left(S^{+}\right)$. 
We proceed similarly in order to obtain a set of initial conditions of the collision orbits belonging to the stable manifold $W^{s}\left(S^{-}\right)$for $H=\mathfrak{H}$ fixed. For each initial condition, we integrate system (11) backward in time.

Concerning the value of $s$, we have carried out several tests taking different values of $s$ (ranging from $10^{-7}$ to $10^{-5}$ ) giving rise to the same results.

(ii) Once we know how to obtain ejection and collision orbits, a second goal is to focus our attention on showing the existence of $n$-EC orbits. For this purpose, we distinguish between two different strategies. Both methods require the computation of the intersection of the flow of system (11) with the Poincaré section $\Sigma: \quad g(\boldsymbol{x})=g(r, \theta, v, u)=v=0$. To obtain numerically such Poincaré section, we remark that given an initial condition $\boldsymbol{x}$ and the associated solution $\boldsymbol{\phi}(t, \boldsymbol{x})$, we apply a Newton's method to obtain a suitable $\tilde{\tau}(\boldsymbol{x})$ such that $g(\phi(\tilde{\tau}(\boldsymbol{x}), \boldsymbol{x}))=0$.

The two strategies to compute $n$-EC orbits are the following Method 1: Intersection of the manifolds $W^{u}\left(S^{+}\right), W^{s}\left(S^{-}\right)$with $\Sigma$.

For a fixed $H$, let us denote $\Sigma_{n}$, the $n$-th crossing with $\Sigma$ and define $D_{n}^{+}=$ $W^{u}\left(S^{+}\right) \cap \Sigma_{n}$, and $D_{n}^{-}=W^{s}\left(S^{-}\right) \cap \Sigma_{n}$. In this first strategy, we look for intersection points belonging to $D_{n}^{+} \cap D_{n}^{-}$. Any such point corresponds to an $n$-EC orbit (from this point on, the solution tends forward (backward) in time to an equilibrium point in $S^{-}\left(S^{+}\right)$.

For example, we take the particular values of $\mu=0.5$ and $H=H_{L_{1}}(0.5)=$ -2.125. In Figure 6 left, $W^{u}\left(S^{+}\right)$and $\left.W^{(} S^{-}\right)$are shown up to $\Sigma_{1}$ in variables $(x, y, v)$, and the right Figure displays the resulting curves $D_{1}^{+}$and $D_{1}^{-}$in $(x, y)$ variables. We can see that both curves intersect at four points which correspond to four 1-EC orbits (for these values of $\mu$ and $H$ ).

At this point two remarks must be made:

1. Due the symmetry (4), we only need to compute $D_{n}^{+}$, and apply the symmetry to obtain $D_{n}^{-}$and then plot the intersection $D_{n}^{+} \cap D_{n}^{-}$.

225 2. The previous approach will be useful and easy for $n=1$, since the curves $D_{n}^{+}$ and $D_{n}^{-}$look as nice near circles (for the ranges of $H$ considered). However for $n \geq 2$, such curves are more intricate and the intersection $D_{n}^{+} \cap D_{n}^{-}$is not so 

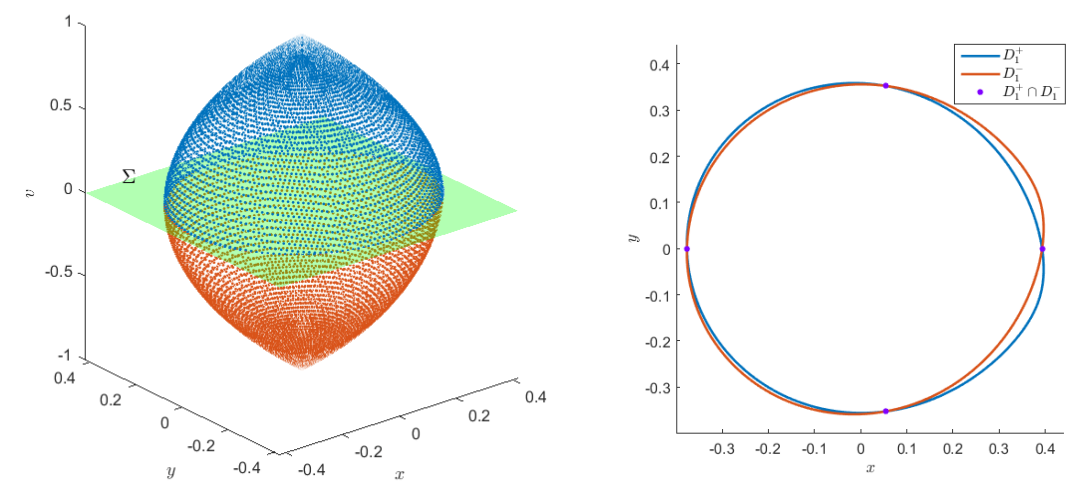

Figure 6: Left. $W^{u}\left(S^{+}\right)$(ejection orbits in blue) and $W^{s}\left(S^{-}\right)$(collision orbits in red) up to $\Sigma_{1}$ for $\mu=0.5$ and $H_{L_{1}}(0.5)$. Right. The associated curves $D_{1}^{+}$and $D_{1}^{-}$.

straightforward. See for example Figure 7 where curve $D_{2}^{+}$is shown for $\mu=0.5$ and different values of $H$.

So, we will apply the following appropriate alternative method.
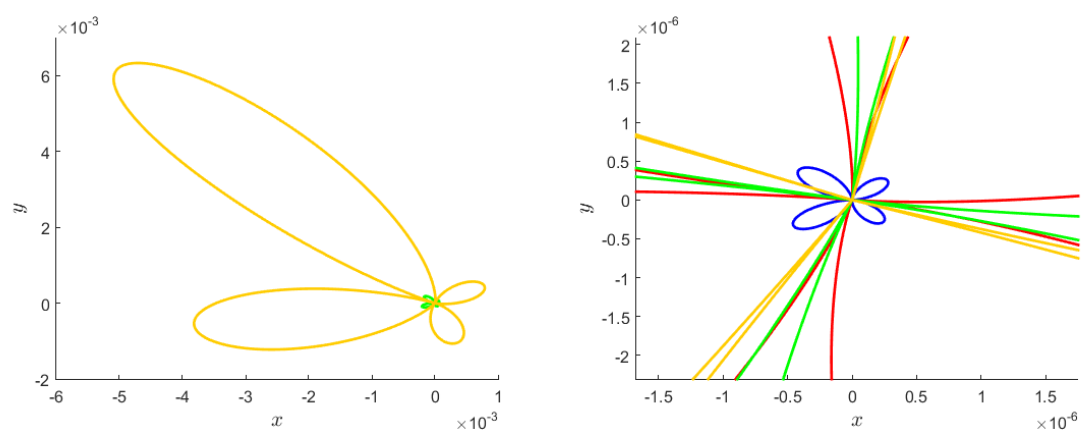

Figure 7: $D_{2}^{+}$in variables $(x, y)$ for $\mu=0.5$ and values of $H-5.25$ in blue, -3.25 in red, -2.75 in green for $H_{L_{1}}(\mu)$ in yellow. On the right: blow up.

\section{Method 2: Singularity in time}

In this method and for fixed $H$, we take the set of initial conditions of ejection orbits, parameterized by $\theta_{0} \in[0,2 \pi]$, we consider the $\Sigma_{2 n}$ intersection of the ejection orbits i.e. $W^{u}\left(S^{+}\right) \cap \Sigma_{2 n}$ (similarly we might consider $W^{s}\left(S^{-}\right) \cap \Sigma_{2 n}$ ) and we look for vertical asymptotes in time $T_{2 n}$ in the curve $\left(\theta_{0}, T_{2 n}\right), \theta_{0} \in[0,2 \pi]$ ( $T_{j}$ being the necessary time to reach $\Sigma$ at the $j$-th crossing). Any value of $\theta_{0}$ 
such that $T_{2 n}$ becomes unbounded will be called a singularity and the associated ejection orbit will be an EC orbit.

For example, for $n=1$, in order to identify the 1-EC orbits, we show in Figure 8 the curve $\left(\theta_{0}, T_{2}\right)$ for $\mu=0.5$ and $H=H_{L_{1}}(0.5)$. We observe four asymptotes, that correspond to four 1-EC orbits in accordance with method 1.

We want to remark that a similar approach is applied to $n$-EC orbits taking into account the curve $\left(\theta_{0}, T_{2 n}\right)$. However, in this curve we have all the singularities (vertical asymptotes in time) corresponding to EC orbits, so in order to detect the actual $n$-EC orbits, we will take into account only the singularities appearing in the curve $\left(\theta_{0}, T_{2 n}\right)$ and not in the curve $\left(\theta_{0}, T_{2 n-2}\right)$, to discard the $j$-EC orbits, for $j=1, \ldots, n-1$. (This will be observed later on in Figure 18).

(iii) Finally a natural question arises: in order to compute a precise 1-EC orbit (or $n$-EC orbit in general), how do we compute the precise value of $\theta_{0}$ (i.e. its initial condition, for $s$ fixed), such that $T_{2}\left(T_{2 n}\right)$ becomes unbounded? The key stone is that, along each U-shaped curve branch of the curve $\left(\theta_{0}, T_{2 n}\right)$ (see Figures 8 and 10), the value of $u$ remains constant in sign and it changes sign consecutively as we change the U-shaped branch, when increasing $\theta_{0} \in[0,2 \pi]$. Precisely at the boundary of each branch $u$ is equal to 0 (since an EC orbit is a heteroclinic orbit connecting two equilibrium points with $u=0$ ). So in order to find a particular 1-EC orbit ( $n$-EC orbit), we simply apply a bisection method on a suitable interval of $\theta_{0}$ such that there is an asymptote in between, i.e. a zero on $u$ at the second crossing with $\Sigma$ (or at the $2 n$-th crossing in general).

To end this Subsection, we point out that we have used double precision throughout all the computations and a Runge-Kutta-Fehlberg 8(7) method in order to integrate numerically the system of ODE given by (11).

\subsection{Results for $1-E C$ orbits}

\subsubsection{Existence of four 1-EC orbits.}

We have considered a grid of values of $\mu$ in the interval $[0.01,0.5]$ and for any given $\mu$ and fixed $H \leq H_{L_{1}}(\mu)$, we have applied method 1, i.e. we have taken a set of initial conditions (parametrized by $\theta_{0} \in[0,2 \pi]$ according to (16)) 
of ejection orbits belonging to $W^{u}\left(S^{+}\right)$, we have computed $D_{1}^{+}=W^{u}\left(S^{+}\right) \cap \Sigma_{1}$ (similarly $\left.D_{1}^{-}\right)$and their intersection $D_{1}^{+} \cap D_{1}^{-}$. Let us denote $\left(r_{0}, \theta_{0}, v_{0}, u_{0}\right)$ (with $u_{0}=0$ ) the initial condition of an ejection orbit, and $\left(r_{i}, \theta_{i}, v_{i}, u_{i}\right)$ the values of $(r, \theta, v, u)$ of this orbit at $\Sigma_{i}(i$-th intersection with $\Sigma$ ).

We have obtained qualitatively the same kind of results for any $\mu$. The numerical simulations carried out confirm not only the analytical results for any $\mu$ and $H$ small enough: existence of four 1-EC orbits, but in addition this result holds true for any $\mu \in(0,0.5]$ and $H \leq H_{L_{1}}(\mu)$.

To show the numerical results obtained, we take, for example, $\mu=0.5$ (for other values of $\mu$ see [16]). In Figure 9 we show the manifolds $W^{u}\left(S^{+}\right)$and $W^{s}\left(S^{-}\right.$) (up to $\Sigma_{1}$ ) for $\mu=0.5$ and different values of $H$ (Figure 9 left). We also plot the corresponding curves $D_{1}^{+}$and $D_{1}^{-}$and the projection of the 1-EC orbits in the $(x, y)$ variables (Figure 9 right). We clearly see four 1-EC orbits for each value of $H$. Concerning the symmetries of these 1-EC orbits, and due to (4), we point out that two of them cross the $x$ axis (i.e. $\theta_{1}=0, \pi$ ) when they intersect with $\Sigma$ (so $\dot{x}=0$ ) and the projection of each of these two orbits in the $(x, y)$ variables is symmetric with respect to the $x$; the $((x, y)$ projection of the $)$ other two EC orbits are non-symmetric but one of them can be obtained from the other one applying the $x$ axis symmetry. See Figure 9 right.

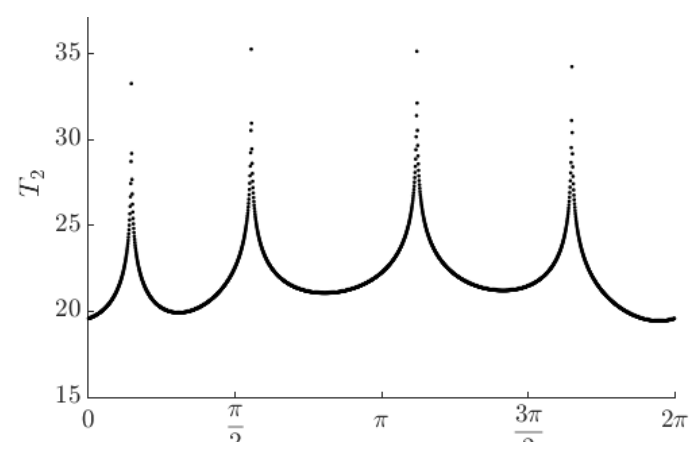

Figure 8: Curve $\left(\theta_{0}, T_{2}\right)$ for $\mu=0.5$ and $H_{L_{1}}(0.5)$.

Now let us describe the results applying method 2 .

In order to show the results obtained with this method, we only need to 

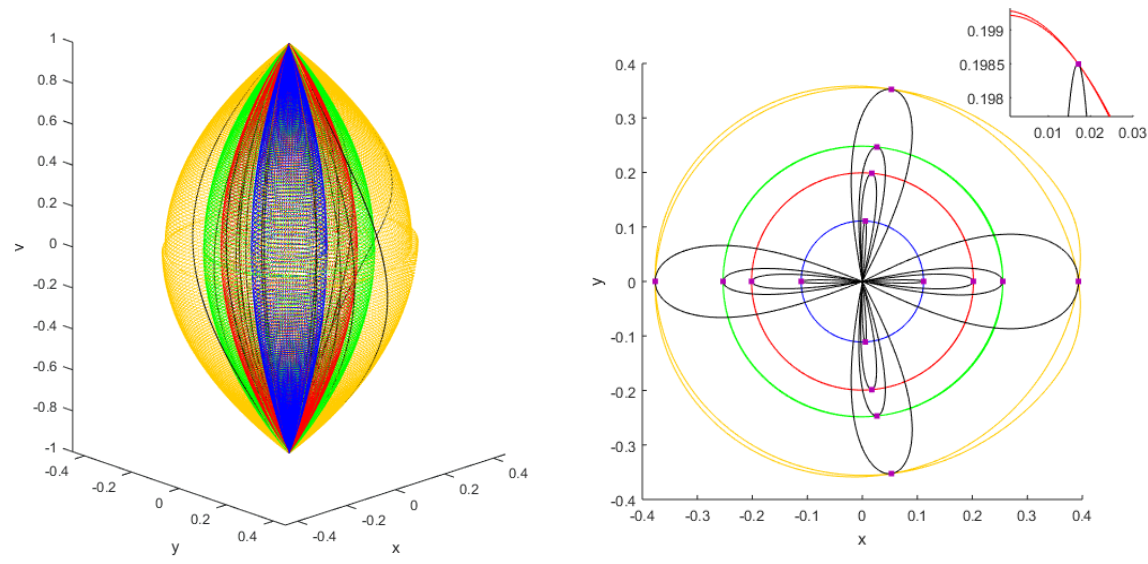

Figure 9: Left. $W^{u}\left(S^{+}\right)$(ejection orbits) and $W^{s}\left(S^{-}\right)$(collision orbits) up to $\Sigma_{1}$ for $\mu=0.5$ and values of $H-5.25$ in blue, -3.25 in red, -2.75 in green and $H_{L_{1}}(\mu)$ in yellow. In black the 1-EC orbits for such values of $H$. Right. Associated $D_{1}^{+}$and $D_{1}^{-}$. In purple the points of the EC orbits at $\Sigma_{1}$ and in black the projections of the EC orbits on the configuration plane $(x, y)$.

compute $T_{i}$ (the necessary time to reach $\Sigma_{i}$ ). In Figure 10 we plot the curves $\left(\theta_{0}, T_{2}\right)$ (left) and $\left(\theta_{1}, T_{2}\right)$ (right) for $\mu=0.5$ and different values of $H$. In accordance with the previous method, from the left plot (using $\left(\theta_{0}, T_{2}\right)$ ) we can conclude that for any $\mu$ and $H$ considered, there are four 1-EC orbits. From the right one, (using $\left(\theta_{1}, T_{2}\right)$ ), we have the additional information about the symmetry of the $(x, y)$ projection of such EC orbits (applying (4)): there are always two 1-EC orbits symmetric with respect to the $x$ axis $\left(\theta_{1}=0, \pi\right)$ and two non symmetric orbits such that one can be obtained from the other one (if one of them has $\theta_{1}=\sigma$ then the other one has $\left.\theta_{1}=2 \pi-\sigma\right)$.

\subsubsection{Families of 1-EC orbits. Bifurcations}

It is clear, from the Hill regions, that for $H<H_{L_{1}}(\mu)$ the ejection motion is restricted to a bounded permissible region around the big primary $m_{1}$ (see

Figure 1). The goal of this Section is to give an insight, concerning EC orbits, 

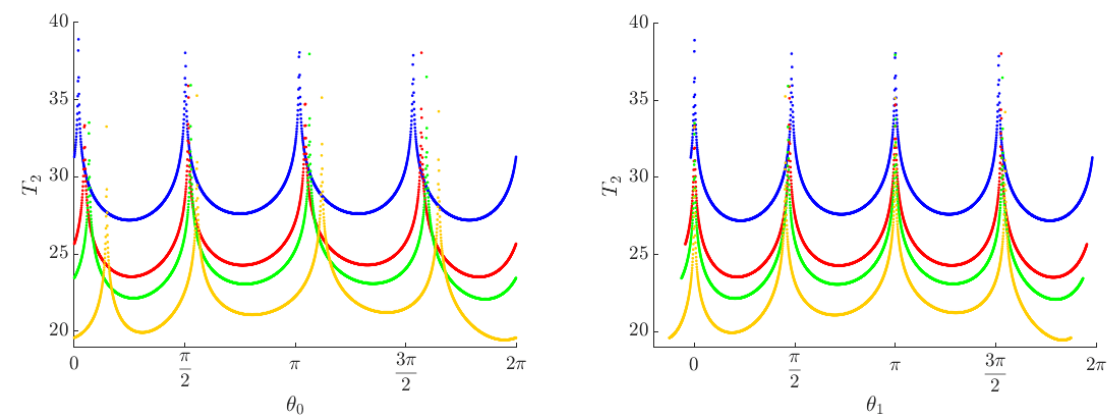

Figure 10: $T_{2}$ as a function of $\theta_{0}$ (left) and $\theta_{1}(t o p)$ and $\mu=0.5$ and values of $H-5.25$ in blue, -3.25 in red, -2.75 in green and $H_{L_{1}}(0.5)$ in yellow.

of the richness of the dynamics that can take place as far as $H \geq H_{L_{1}}(\mu)$, due to not only the presence of both primaries, but also the chaotic involved dynamics because of the existence of unstable periodic orbits and their invariant manifolds (just take into account the Lyapunov periodic orbits for instance, see [3] and references therein). In particular, new bifurcating families of 1-EC orbits appear.

So applying method 2 we do a massive simulation in order to detect the four families of 1-EC orbits, when varying $H \leq H_{L_{2}}(\mu)$ for any given $\mu \in(0,0.5]$. We call such families $\alpha, \beta, \gamma$ and $\delta$. The results are shown in Figure 11 for $\mu=0.5$. We call diagram $\left(\theta_{0}, H, T_{2}\right)$, the plot where we obtain $T_{2}$ as a function of the angle $\theta_{0}$ and the energy $H$. For a fixed $H$, the four angles $\theta_{0}$ for which $T_{2}$ is large, correspond to the four particular values of $\theta_{0}$ for which there exist 1-EC orbits.

We remark from this Figure, that for $H_{L_{1}}(\mu)<H$ there appear new bifurcated families (although difficult to be seen in the Figure). Let us describe this phenomenon for $\mu=0.5$ and $-2.125=H_{L_{1}}(0.5)<H<H_{L_{2}}(0.5)=$ -1.853398112043077 .

In Figure 12 we plot a zoom in a neighbourhood of the $\alpha$ family. We observe apparently a new family which we call $\eta$. But before describing this bifurcation, we remark three different regions, labelled by $A, B$ and $C$. First of all, we see 


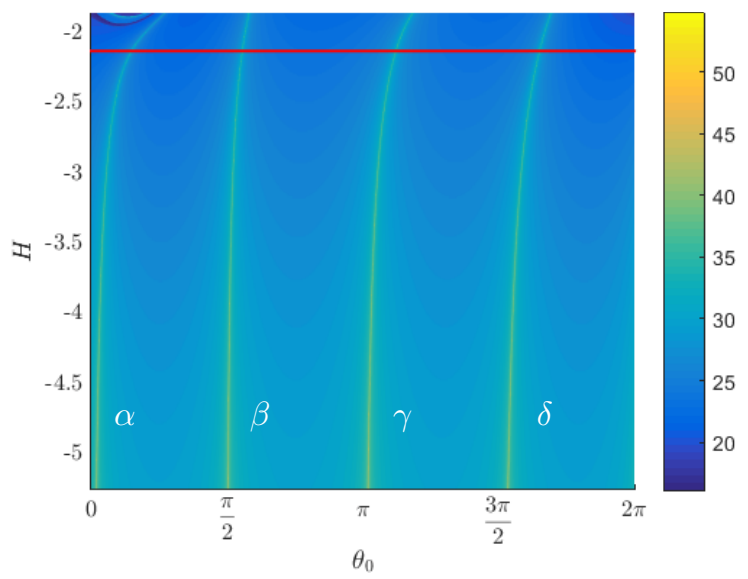

Figure 11: Diagram $\left(\theta_{0}, H, T_{2}\right)$ for $\mu=0.5$ for values of $H \leq H_{L_{2}}(0.5)$. The red line corresponds to $H_{L_{1}}(0.5)$.
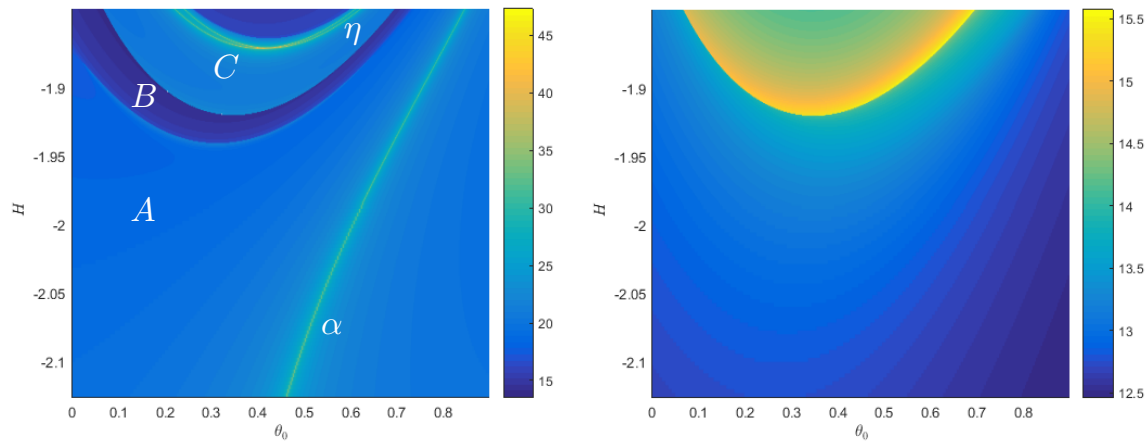

Figure 12: Zoom of diagram $\left(\theta_{0}, H, T_{2}\right)$ (left) and $\left(\theta_{0}, H, T_{1}\right)$ (right) for $\mu=0.5$

that the ejection orbits that live in $A$ and $C$ have a similar value of $T_{2}$, but a smaller one for the region $B$ in between.

The behaviour of the orbits in these regions is the following (see Figure 13): orbits in region $A$ do not have enough energy to go to the small primary and they behave in a usual way, starting at ejection with the big primary, having a loop and returning close to it (see orbit red in the Figure). Those orbits in region $B$ (in blue) do have enough energy to go close to the small primary, but not enough energy to go in a direct path, they describe a small loop before getting there. Just short after the loop they reach $T_{2}$ and that is the reason why 

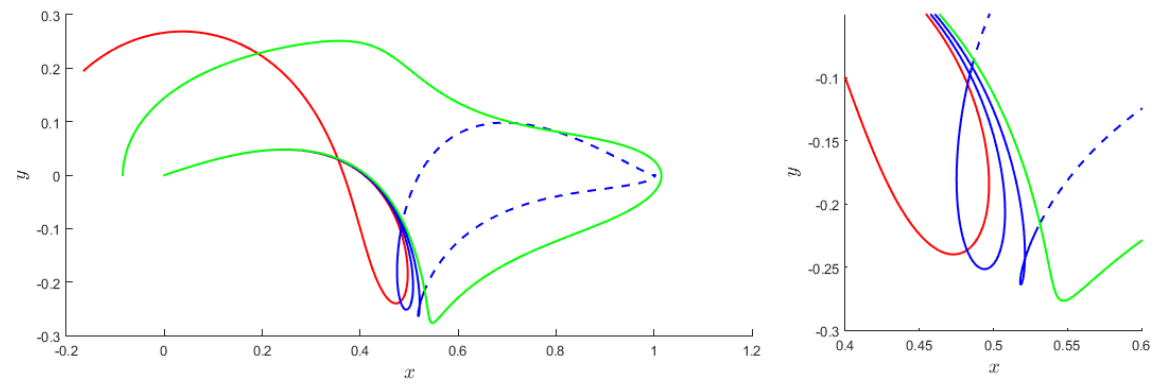

Figure 13: Different ejection orbits belonging to region $A$ (red), $B$ (blue) and $C$ (green) for $\theta_{0}=0.4$ (left) and a zoom around the turning point of the same orbits (right). All of them have a continuous path for time up to $T_{2}$ and a discontinuous path only for those orbits in region $B$ and along the range of time from $T_{2}$ up to $T_{3}$.

$T_{2}$ is smaller in the region $B$ (see the continuous small loop of the two orbits in blue in Figure 13). Finally those orbits in region $C$ have enough energy to visit the small primary and return back (close) to the big one, along the time $T_{2}$ (see the long green orbit in the Figure).

Of course, this behaviour for the orbits is reflected in the diagram $\left(\theta_{0}, H, T_{1}\right)$, see Figure 12 right, where the boundary between regions $B$ and $C$ is clearly seen. Along the yellow region in the Figure 12 right we observe how $T_{1}$ is sensitively bigger since for this region, the loop does not exist, the orbits visit a neighbourhood of the small primary $m_{2}$ and they need a larger time to reach $\Sigma$. See also Figure 13.
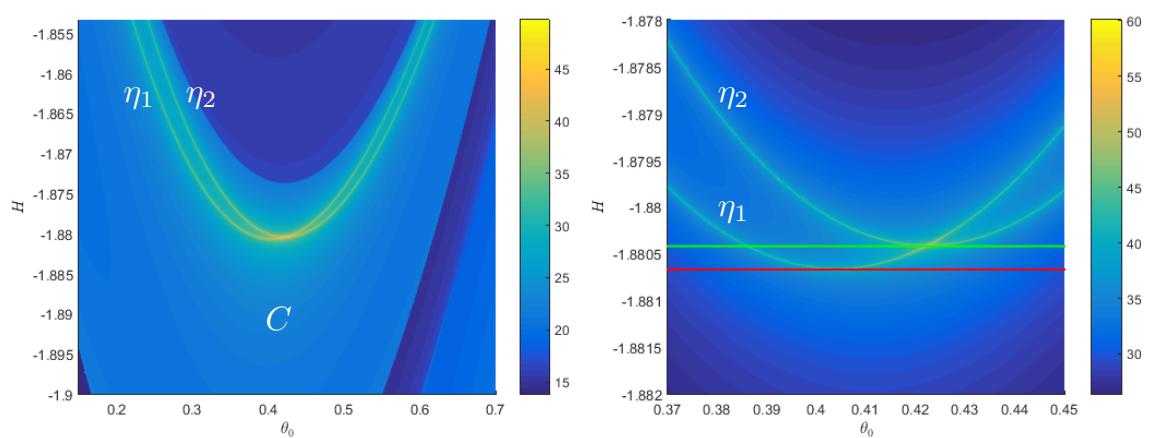

Figure 14: Zoom (of Figure 12) of the new bifurcated families. Red and green horizontal lines correspond to $H=H_{b 1}$ and $H=H_{b 2}$ respectively. 
Let us focus now on the bifurcated family $\eta$ (see Figure 12). These new EC orbits detected correspond to orbits that visit the region close to the small primary at his first intersection with $\Sigma$. If we zoom this region (see Figure 14) around $\eta$, we observe actually two different curves, that is two different bifurcated families, which we label $\eta_{1}$ and $\eta_{2}$ : when increasing $H$, the first family that appears is $\eta_{1}$, at a particular minimum value $H=H_{b 1}$. As far as $h$ increases, a new branching point appears at $H=H_{b 2}$, and we obtain a new family labelled $\eta_{2}$. 1-EC orbits belonging to $\eta_{1}$ are symmetric with respect to the $x$ axis. In Figure 15 left, we plot the EC orbit (in blue) for $H_{b 1}$ where $\eta_{1}$ is born, and in red and in green the two corresponding 1-EC orbits of family $\eta_{1}$ for two increasing fixed values of $H$. 1-EC orbits belonging to $\eta_{2}$ are non symmetric. In Figure 15 right, we plot the EC orbit (in blue) for $H_{b 2}$ that belongs to $\eta_{1}$ and where $\eta_{2}$ is born, and in red and in green the two corresponding 1-EC orbits of family $\eta_{2}$ for two increasing fixed values of $H$.
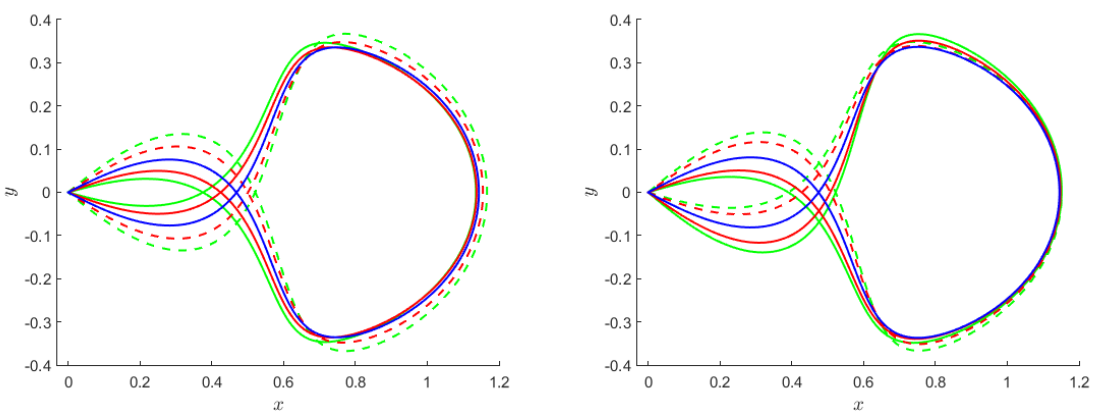

Figure 15: 1-EC bifurcated orbits belonging to $\eta_{1}$ (left, for $H=H_{b 1}$ in blue, for a value $H>H_{b 1}$ in green and in red) and $\eta_{2}$ (right, for $H=H_{b 2}$ in blue, for a value $H>H_{b 2}$ in green and in red).

Therefore we can conclude that the existence of only four 1-EC orbits is no longer true for higher values of the energy $H$, since there appear new ones. We plot in Figure 16 eight 1-EC orbits for $\mu=0.5$ and $H_{L_{2}}(0.5)$, together with the Hill's region.

A final remark is that the bifurcation described for $\mu=0.5$ also may take place for other small values of $\mu$ and less restrictive Hill's regions (higher values 

rich dynamics starting at ejection with the big primary takes place. But this is a more intricate study which will be done in a next future.

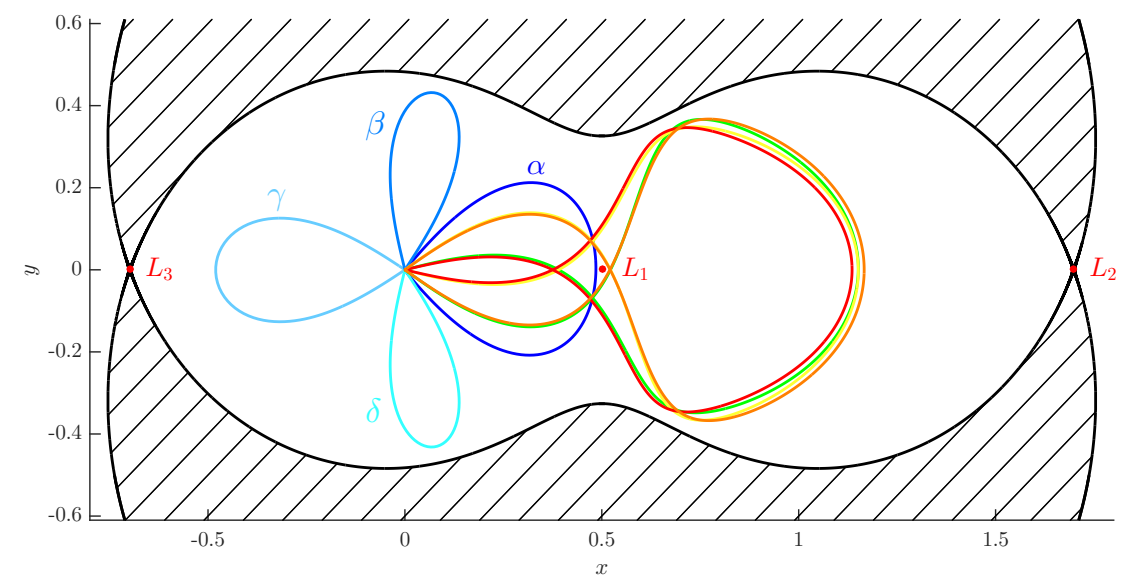

Figure 16: $(x, y)$ projection of the eight 1-EC orbits that exist for $\mu=0.5$ and $H_{L_{2}}(\mu)$. In blue the known ones and in other colours the new bifurcated orbits.

\subsection{Results for $n$-EC orbits. Families and bifurcations.}

Recall that an $n$-ejection-collision orbit is an orbit which ejects from the

365 first primary and collides again after the distance to this primary has reached $n$ times a relative maximum (see Figure 5). As stated in section 4.1 describing the numerical methodology, method 2 is more suitable to study $n$-EC orbits.

We have done massive simulations applying method 2 for any value of $\mu \in$ $[0.01,0.5]$ and for a fixed $\mu$, we have considered energy levels below $H_{L_{1}}(\mu)$ (in order to avoid collision with the second primary; since we take long time integration of the equations, the influence of the second primary makes the dynamics more and more intricate for increasing values of $n$ ). The results are qualitatively similar for any value of $\mu$.

The numerical results obtained show that for all $\mu \in(0,0.5]$ and all $n$ there exists an $\hat{H}(\mu, n)$ such that for $H \leq \hat{H}(\mu, n)$ there exist four $n$-ejection-collision 
orbits, which can be characterized in a way similar to the characterization of the 1-ejection-collision orbits:

- There are two of them which intersect the $x$-axis (i.e. $\theta_{n}=0, \pi$ ) when they intersect the Poincaré section $\Sigma_{n}$. They are symmetric with respect to the $x$ axis (taking the $(x, y)$ projection). We will follow the previously used notation and call the corresponding families (when varying $H$ ) $\alpha_{n}$ and $\gamma_{n}$ respectively.

- The other two (which do not intersect the $x$-axis when they cross the Poincaré section $\Sigma_{n}$ ) are non symmetric with respect to the $x$-axis (taking the $(x, y)$ projection), but one can be obtained from the other one applying symmetry (4). Following again the notation previously introduced we will call the corresponding families $\beta_{n}$ and $\delta_{n}$, respectively.

We show examples of orbits belonging to families $\alpha_{n}, \beta_{n}, \gamma_{n}$ and $\delta_{n}$, for $n=2$ and $n=3$ in Figure 17 for $\mu=0.1$ and different values of $H$.

In order to show the families of $n$-EC orbits, for $n \geq 2$, when varying $H$, we will use the diagram $\left(\theta_{0}, H, T_{2 n}\right)$ as we did for $n=1$. However, we must take into account, that the diagram $\left(\theta_{0}, H, T_{2 n}\right)$, contains the families of $n$-EC orbits and the families of $1-\mathrm{EC}, \ldots,(n-1)$-EC orbits as well. Therefore to detect the actual families of $n$-EC orbits, we must take into account the new singularities that do not appear in the diagrams $\left(\theta_{0}, H, T_{2 j}\right)$, for $j=1, \ldots, n-1$. In Figure 18 , we take $\mu=0.1$ and we show the diagrams $\left(\theta_{0}, H, T_{2 n}\right)$, for $n=1, \ldots ., 5$ and $H \leq H_{L_{1}}(0.1)=-1.843476614939948$. Thus, we observe that for each $n=1, \ldots, 5$, there exist four families of $2, \ldots ., 5$-EC orbits, taking as parameter the value of $H$ up to a suitable one, $H \leq \hat{H}(\mu, n)$.

However, for high values of $n$ and $H$, the statement about the existence of four families of $n$-EC orbits can no longer be true because of the influence of the other primary and a rich dynamics. We will focus on two particular phenomena: (i) the intersection of families and (ii) bifurcation of families.

Concerning (i), if we consider the new singularities obtained at the $2 n$-th intersection with $\Sigma$, i.e. the initial values $\theta_{0}$ of the $n$-EC orbits (giving rise to the 

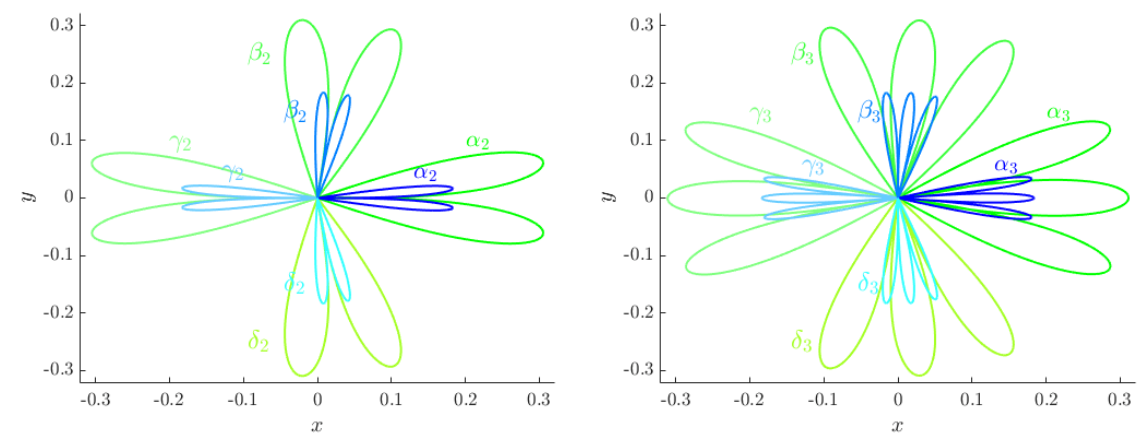

Figure 17: The four $n$-ejection-collision orbits for $\mu=0.1$ and $n=2$ (left) and $n=3$ (right) for $H=-5.05$ (blue) and $H=-3.05$ (green)

yellow curves in the $\left(\theta_{0}, h, T_{2 n}\right)$ diagram), and these singularities intersect some of the previously obtained ones, i.e. singularities in the $k$-th intersection with $\Sigma$ for $k<2 n$, then there are no $n$-EC orbits because, for those corresponding values of $\theta_{0}$, the orbit has already had a previous collision. This phenomenon can be seen with more detail in Figure 19, where the colour of the $T_{10}$ variable has been rescaled in the amplification for easier viewing.

Concerning (ii), we observe, when increasing $H$, the termination of families or collapse of two different families on to a singularity belonging to another different family. This is the situation shown in Figure 20 for $\mu=0.1$, where the two families of non-symmetric EC orbits, $\beta_{4}$ and $\delta_{4}$ collapse on to a symmetric EC belonging to family $\gamma_{4}$. In other words, if we consider the families when decreasing the $H$ parameter, we would say that the two families $\beta_{4}$ and $\delta_{4}$ have bifurcated from the family $\gamma_{4}$ at the collapsing orbit.

Finally, there is another type of bifurcation when increasing the $H$ parameter and it is related to the appearance of new bifurcating families from the main four families. This is the same type of bifurcation mentioned in the case of 1-EC families. For example, we show in Figure 21, the new bifurcating families $\alpha_{4}^{1}, \alpha_{4}^{2}$ of non-symmetric 4-EC orbits from family $\alpha_{4}$, for $\mu=0.1$ (we show a zoomed diagram $\left(\theta_{0}, H, T_{8}\right)$ from the one in Figure 18, as well as the bifurcating orbit and two orbits of each family for different values of $H$ ). 


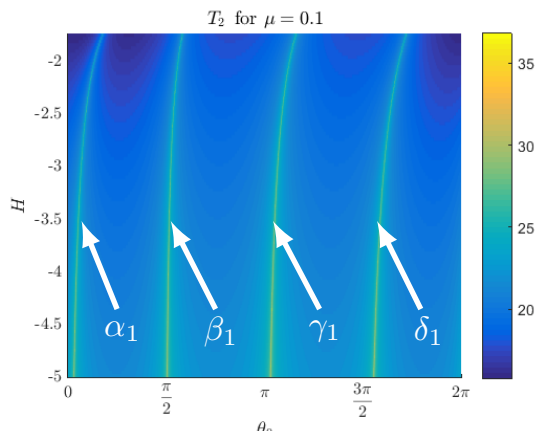

(a)

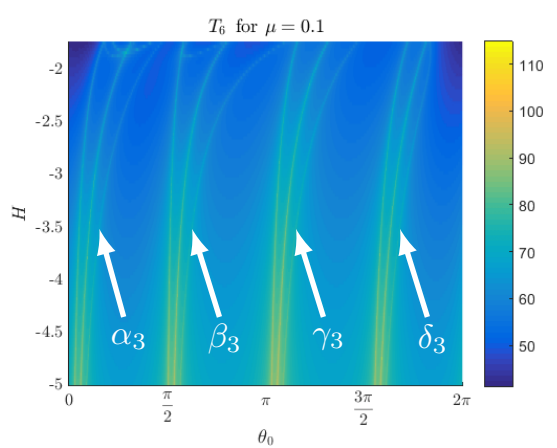

(c)

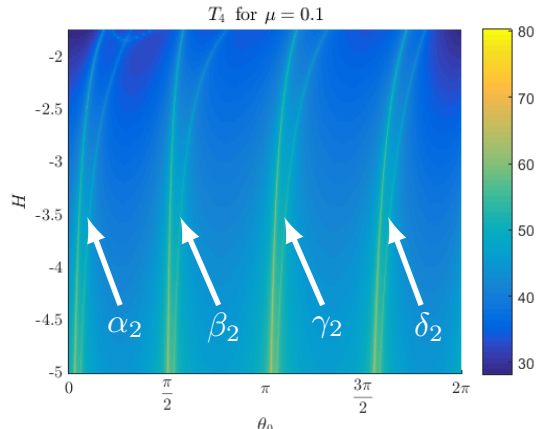

(b)

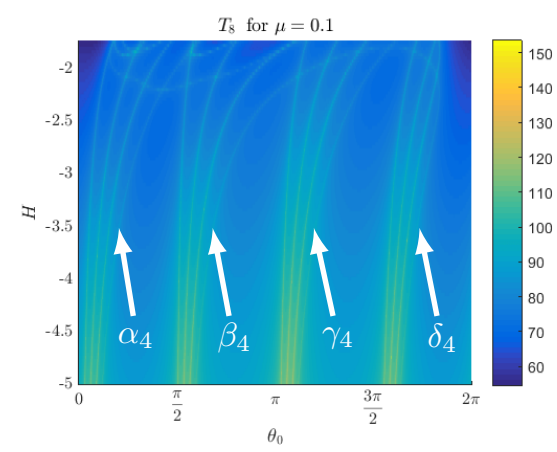

(d)

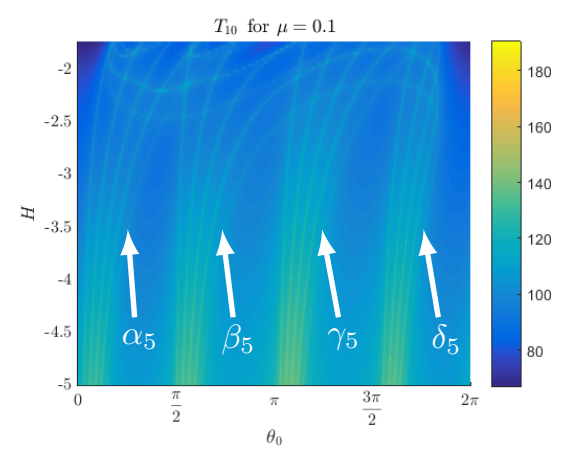

(e)

Figure 18: From left to right and top to bottom values of $T_{2 i}$ for $i=, 1 \ldots, 5$ as a function of $\theta_{0}$ and $H$ for $\mu=0.1$ 


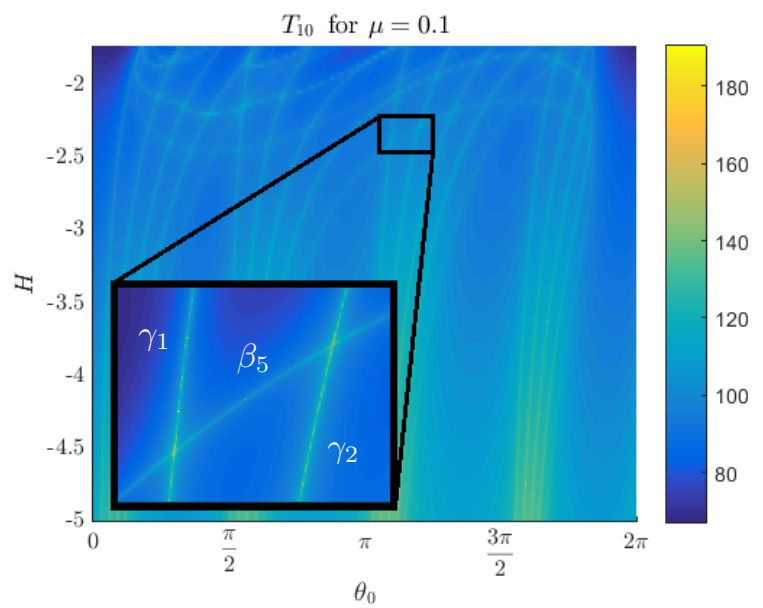

Figure 19: Zoom of the diagram $\left(\theta_{0}, H, T_{10}\right)$, for $\mu=0.1$ with the intersection of singularities.

\section{Conclusions}

Concerning previous references on the RTBP, only the analytical proof of the existence of four 1-EC orbits was known for $\mu$ given and very restrictive values of the energy $H(H=h<0$ and $|h|$ big enough). However we do not find, in the literature, any systematic numerical computation of EC orbits. In this paper, we have presented a numerical methodology to study and compute not only 1 - EC orbits but the generalization to the $n$-EC orbits for any $n \geq 1$, significantly extended ranges of the energy values and for any value of the mass parameter.

The transformation to a regularized system of ODE (to deal with the singularity collision with one of the primaries) as well as the description of the collision manifold provide the suitable setting in order to compute EC orbits regarded as heteroclinic orbits. For their computation we have disregarded the natural (and usual) method that consists of the intersection of the manifolds with a given Poincaré section; since the visualization of such intersections is too intricate, we have described and used another method instead (based on singularities in time, and that provides an easier and clearer view).

We have done massive simulations and obtained families of $n$-EC orbits (for 

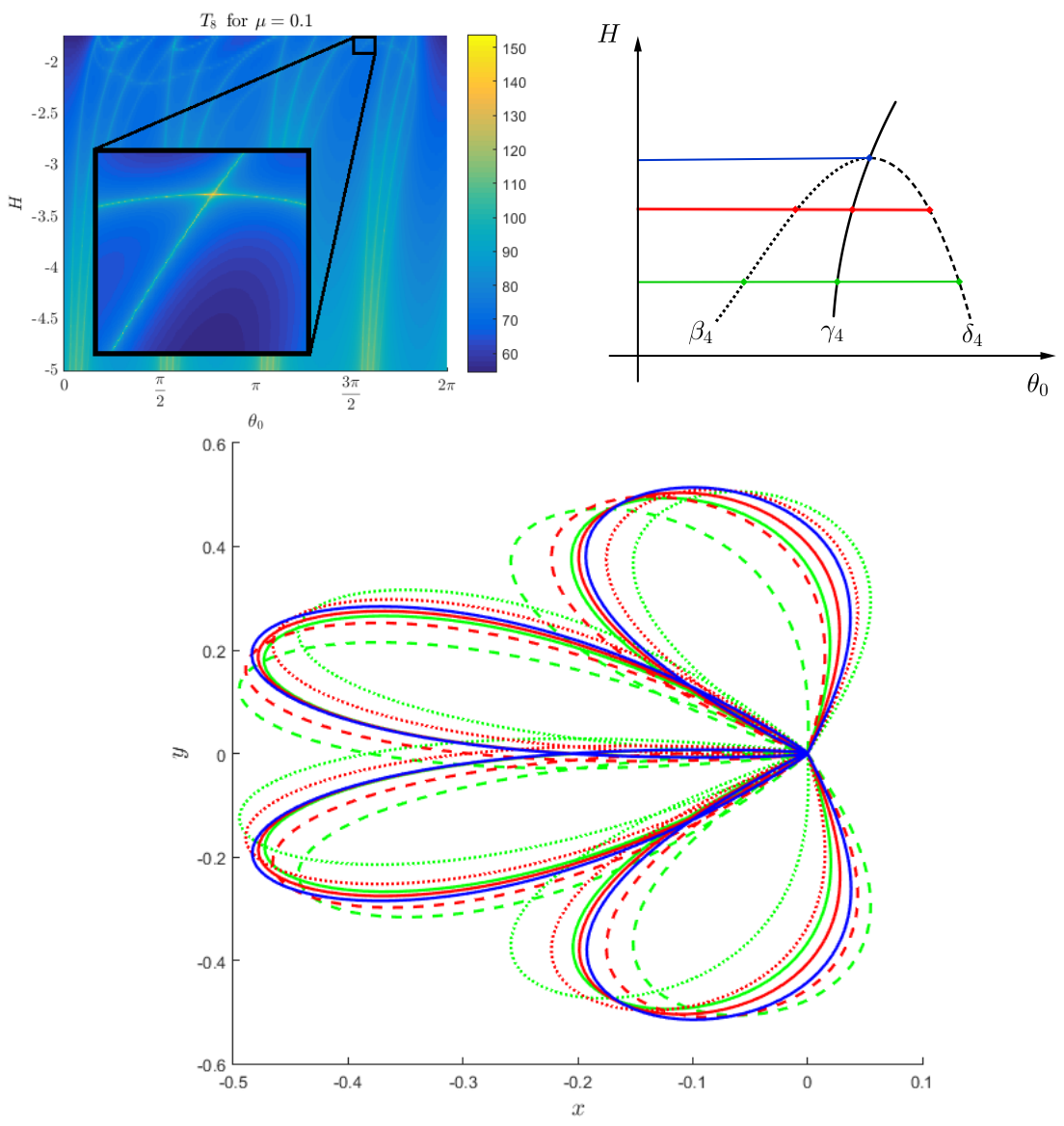

Figure 20: Collapse of families $\beta_{4}$ and $\delta_{4}$ on to an orbit belonging to family $\gamma_{4}$, for $\mu=0.1$. Top left. An amplification of diagram $\left(\theta_{0}, H, T_{8}\right)$. Top right. Qualitative plot in $\left(\theta_{0}, H\right)$ variables. We display the collapsing value of $H$ in blue, and two less energetic values in red and green. Bottom. The orbits themselves. The one belonging to family $\gamma_{4}$ in blue, and the four bifurcated orbits for higher values of $H$ in red and green.

$n \leq 10$ ) when varying the energy (and $\mu$ fixed). Not only have the main four

families been found (as was expected in the particular case $n=1$ ), but also new families have been computed and the discussion of the bifurcations that appear has also been analysed.

Finally we remark that we have obtained similar results for any value of the mass parameter. 

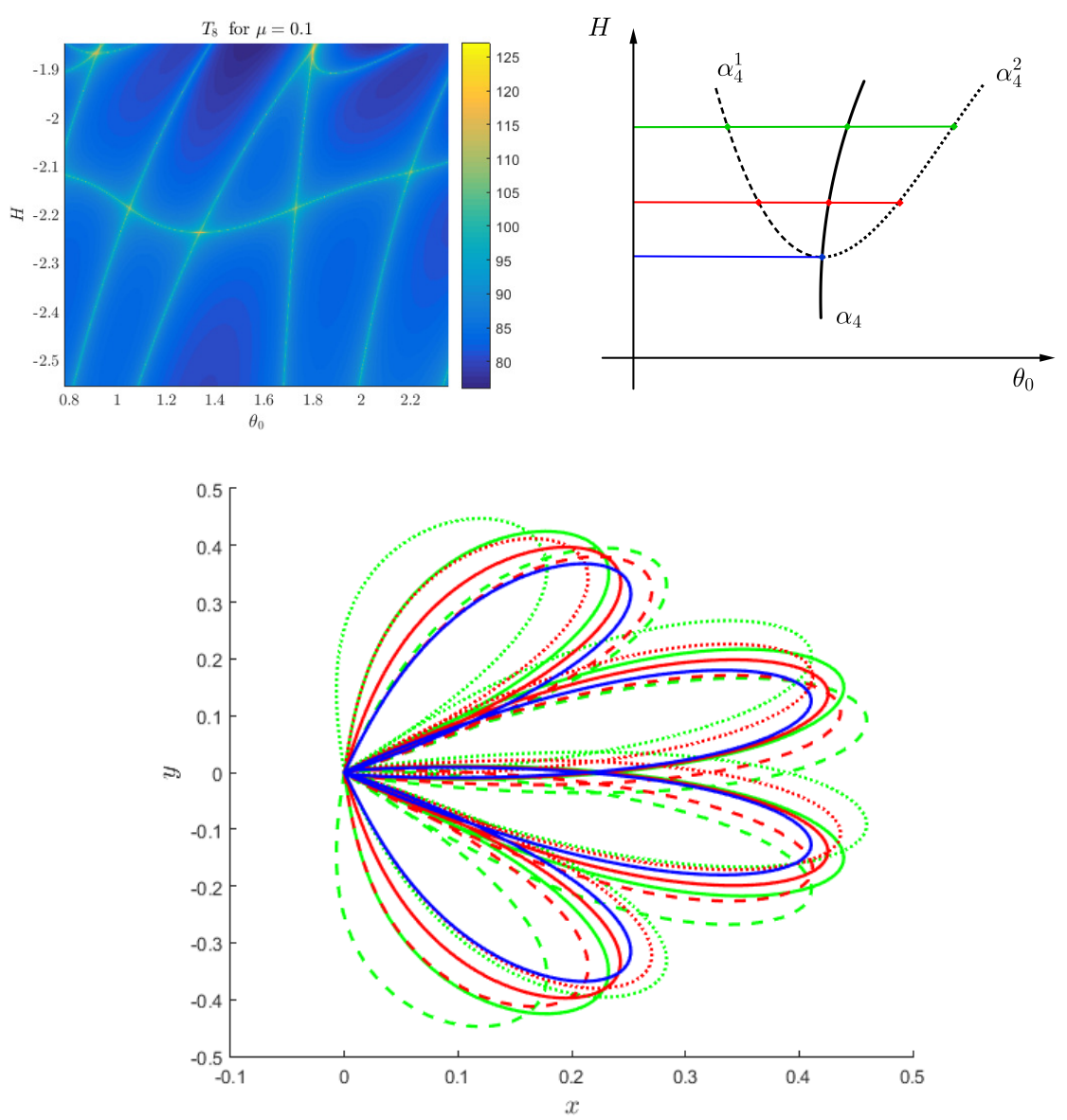

Figure 21: Bifurcation of 4-EC orbits for $\mu=0.1$. Top left. An amplification of diagram $\left(\theta_{0}, H, T_{8}\right)$. Top right. Qualitative plot of the bifurcating families in $\left(\theta_{0}, H\right)$ variables: dotted and discontinuous curves bifurcating from family $\alpha_{4}$ (continuous curve). We display the limit value of $H$ at the bifurcation in blue, and two more energetic values in red and green. Bottom. The orbits themselves. The one belonging to family $\alpha_{4}$ in blue, and the four bifurcated orbits for higher values of $H$ in red and green.

\section{Acknowledgments}

M. Ollé and O. Rodríguez have been supported by the Spanish MINECO/FEDER grant MTM2015-65715-P and the Catalan grant 2014SGR-00504. J. Soler has been supported by MINECO/FEDER grant number MTM2016-77278-P. 


\section{References}

[1] Astakhov, S. A., Burbanks, A. D., Wiggins, S., Farrelly, D. Chaos-assisted capture of irregular moons. Lett. to Nature 2003;423:264-267.

[2] Astakhov, S. A., Lee, E. A., Farrelly, D. Formation of Kuiper-belt binaries through multiple chaotic scattering encounters with low-mass intruders. Mon. Not. R. Astron. Soc. 2005;360:401-415.

[3] Barrabés, E., Mondelo, J. M., Ollé, M. Numerical continuation of families of homoclinic connections of periodic orbits in the RTBP. Nonlinearity 2009;22:2901-2918.

[4] Bozis, G. Sets of collision periodic orbits in the Restricted problem. In: Periodic orbits, stability and resonances, G.E.O. Giacaglia (eds). Holland: D. Reidel Pub. Co.; 1970, p. 176-191.

[5] Brunello, A. F.,Uzer, T., Farrelly, D. Hydrogen atom in circularly polarized microwaves: Chaotic ionization via core scattering. Phys Rev A $1997 ; 55: 3730-3745$.

[6] Chenciner, A and Llibre, J. A note on the existence of invariant punctured tori in the planar circular RTBP. Ergod. Th. \& Dynam. Sys. 1988;8:63-72.

[7] Hénon, M. Exploration numérique du problème restreint I. Masses égales, Orbites périodiques. Ann Astrophys 1965;28:499-511.

[8] Hénon, M. Numerical exploration of the Restricted Problem V. Hill's case: Periodic orbits and Their Stability. Astron Astrophys 1969;1:223-238.

[9] Lacomba, E. A. and Llibre, J. Transversal Ejection-Collision Orbits for the Restricted Problem and the Hill's Problem with Applications. J Differ Equations 1988;74:69-85.

[10] Llibre, J. On the Restricted Three-Body Problem when the Mass Parameter is Small. Celestial Mech Dynam Astronom 1982;28:83-105. 
[11] Llibre, J, and Martinez-Alfaro, J. Ejection and collision orbits of the spatial RTBP. Celestial Mech 1985;35:113-128.

[12] Llibre, J. and Pinyol, C. On the Elliptic Restricted Three-Body Problem. Celestial Mech Dynam Astronom 1990;48:319-345.

[10] McGehee, R. Triple Collision in the Collinear Three-Body Problem. Invent Math 1974;27:191-227.

[13] Devaney, R. L. Singularities in Classical Celestial Mechanics. In: Ergodic Theory and Dynamical Systems I, Proceedings Special year, Maryland 1979-80, A. Katok (Ed.), p. 211-333.

[14] Ollé, M. To and fro motion for the hydrogen atom in a circularly polarized microwave field. To appear in Commun Nonlinear Sci Numer Simulat.

[15] Pinyol, C. Ejection-collision orbits with the more massive primary in the planar elliptic restricted three-body problem. Celestial Mech Dynam. Astronom 1995;61:315-331.

[16] Rodríguez, O. Òrbites d'ejecció-col.lisió en el problema restringit de tres cossos. Master Thesis. Universitat Politècnica de Catalunya, 2016.

[17] Sanchez, D.P., and Scheeres, D.J. DEM simulation of rotation-induced reshaping and disruption of rubble-pile asteroids. Icarus, 218, 876-894 (2012).

[18] Stiefel, E. L., Scheifele, G. Linear and regular Celestial Mechanics. Springer-verlag, New York; 1971.

500 [19] Szebehely, V. Theory of orbits. Academy Press, Inc., New York; 1967. 